\title{
Study on Lubricant Liquid Film Performances for Groove-pores Coupled Mechanical Seals
}

\author{
Penggao Zhang ${ }^{a^{*}}$, Long $\mathrm{Wei}^{\mathrm{b}}$
}

Fluid Sealing Measurement and Control Engineering Research and Development Centre of Jiangsu, Nanjing Polytechnic Institute, Nanjing 210048, China

a249954371@163.com, bweilong1972@163.com

Keywords: mechanical seal; groove-pores coupled; lubricant liquid film; numerical simulation

Abstract. The performances of lubricant liquid film of groove-pores coupled mechanical seal are numerical simulated by Fluent, and the effects of depth of groove and pore on liquid film performances are analyzed. The results indicate that dynamic pressure generated by groove-pores coupled mechanical seal is higher than spiral groove mechanical seal, and the highest pressure is at the root of spiral groove. The film pressure, the opening force and the leakage increase first and then decrease with the increases of the depth of groove and pore. The higher ratio of stiffness to leakage is the model of $h_{g}=4 \mu m$ when $p>1 M P a$ and $n<2300 r / m i n$. However, The higher ratio of stiffness to leakage is the model of $h_{g}=20 \mu \mathrm{m}$ when $p<1 M P a$ and $n>2300 \mathrm{r} / \mathrm{min}$.

\section{Introduction}

The spiral groove mechanical seal has a good upstream pumping capacity, and the micro-pores mechanical seal has an obvious dynamic pressure effect ${ }^{[1-2]}$. Groove-pores coupled mechanical seal is feasible for obtaining zero leakage in theory ${ }^{[3-5]}$. The characteristic parameters of groove-pores coupled mechanical seal are calculated and the effects of depth of groove and pore on liquid film properties are analyzed in this paper.

\section{Numerical simulation of Lubricant Liquid Film Performances for Groove-pores Coupled Mechanical Seals}

Basic Assumptions. In view of the complexities of the real operating conditions and calculation, the assumptions are: (1)The sealant is a Newtonian fluid. (2)The friction heat is neglected. (3)The influences of vibration and disturbance of system are neglected. (4) Sliding between liquid and sealing faces is neglected. (5) Inertial force and volume force are neglected.

Geometric Model of Lubricant Liquid Film and Meshing. The structural parameters are listed in table 1, and the lubricant liquid film is shown in Fig.1. The model is enlarged 1000 times in the direction of thickness for describing the lubricant liquid film clearly.

Table 1 Structural parameters of groove-pores coupled mechanical seal

\begin{tabular}{|c|c|c|c|c|c|c|c|c|c|}
\hline $\begin{array}{c}\text { Outer radius } \\
r_{0} / \mathrm{mm}\end{array}$ & $\begin{array}{c}\text { Inner radius } \\
r_{i} / m m\end{array}$ & $\begin{array}{l}\text { Helical angle } \\
\alpha / r a d\end{array}$ & $\begin{array}{l}\text { Ratio of } \\
\text { groove } \\
\text { width } \\
/ B\end{array}$ & $\begin{array}{l}\text { Ratio of } \\
\text { groove } \\
\text { length } \\
/ \gamma\end{array}$ & $\begin{array}{c}\text { Number of } \\
\text { groove } \\
\text { s } \\
/ N_{g}\end{array}$ & $\begin{array}{l}\text { Depth of } \\
\text { groove } \\
h_{g} / \mu m\end{array}$ & $\begin{array}{r}\text { Radius of } \\
\text { pore } \\
r_{p} / m m\end{array}$ & $\begin{array}{c}\text { Number } \\
\text { of } \\
\text { pores } \\
/ N_{p}\end{array}$ & $\begin{array}{l}\text { Thickness } \\
\text { of film } \\
h_{0} / \mu m\end{array}$ \\
\hline 41 & 31 & 0.3 & 0.5 & 0.6 & 12 & 8 & 0.2 & 120 & 4 \\
\hline
\end{tabular}

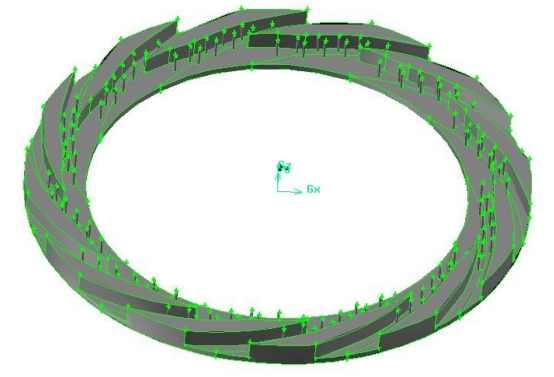

Fig.1 Lubricant liquid film of groove-pores coupled mechanical seal 
Because of symmetric and periodic distribution of grooves and pores, $1 / N_{g}$ film is selected as study objective (see Fig.2). The grid is meshed by Gambit. The study objective is divided into Film 1, 2, 3, groove and $N_{p} / N_{\mathrm{g}}$ column regions. The end faces are meshed by quad element and pave type respectively, and then the surfaces are pulled to hexahedral meshes which the interval between layers is $0.001 \mathrm{~mm}$ by Cooper. The grid is shown in Fig.3, and there are 870161 nodes.

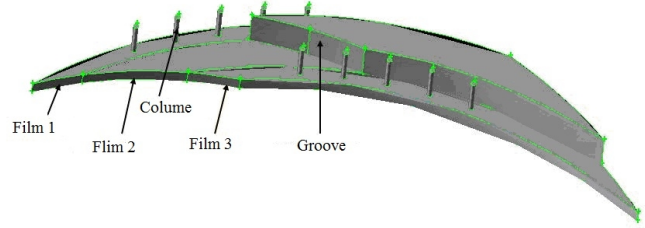

Fig.2 Study objective

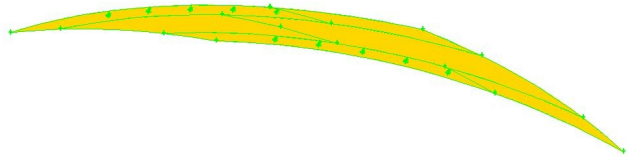

Fig.3 Grid of geometric model

Sealant. The sealant is $20^{\circ} \mathrm{C}$ water, and the thermophysical properties of sealant and operating parameters of testing are seen in Table 2.

Table 2 Thermophysical properties of sealant and operating parameters of testing

\begin{tabular}{ccccc}
\hline \multirow{2}{*}{ Sealant } & $\begin{array}{c}\text { Density } \\
\rho /\left(\mathrm{kg} / \mathrm{m}^{3}\right)\end{array}$ & $\begin{array}{c}\text { Chamber } \\
\text { pressure } \\
p / M P a\end{array}$ & $\begin{array}{c}\text { Ambient pressure } \\
p_{0} / \mathrm{MPa}\end{array}$ & $\begin{array}{c}\text { speed } \\
n /(\mathrm{r} / \mathrm{min})\end{array}$ \\
\hline Water & 998.2 & 0.6 & 0.1 & 3000 \\
\hline
\end{tabular}

Boundary Conditions. The pressure at the outer diameter is higher, the pressure at the inner diameter is lower, and fluid flows from outer diameter to inner diameter because of pressure difference. Boundary conditions of pressure inlet, pressure outlet, and fluid rotating speed are given. Two sealing faces are simulated by no sliding condition. The flow near the sealing face is simulated by the standard wall function method. The mutual motions between fluid and rotating and stationary ring are simulated by the reference frame method.

Solution Method. In this paper, the steady pressure-based implicit solver is chose. Pressure-velocity coupling method is SIMPLEC algorithm. Pressure difference scheme is the second order differential form. Discrete Scheme of diffusion term is central difference scheme. Discrete Scheme of convective term is second-order upwind scheme.

Results. The pressure distribution of groove-pore coupled mechanical seal calculated by Fluent is shown in Fig.4. Furthermore, it can be read from Fluent that the leakage and opening force are $2.41 \mathrm{~L} / \mathrm{h}$ and $1.618 \mathrm{kN}$ respectively. It can be seen in Fig. 4 that the maximum pressure is $1.51 \mathrm{MPa}$, which is 2 times higher than medium pressure, and is higher $0.6 \mathrm{MPa}$ than spiral groove mechanical seal in [6]. So higher dynamic pressure can be obtained in groove-pores coupled mechanical seal, and the maximum pressure appears at the root of spiral groove.
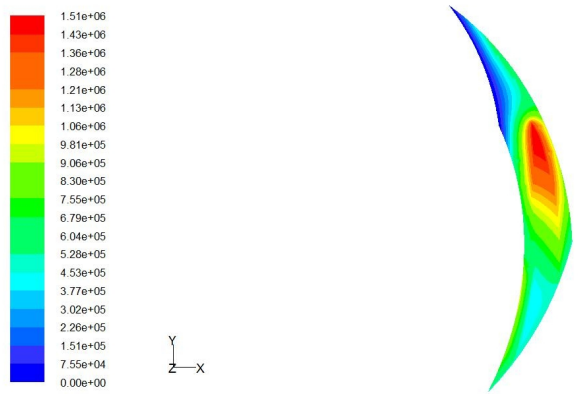

Fig.4 Distribution of pressure of liquid film

Effects of depth of groove and pore on liquid film properties. Effects of depth of groove and pore on liquid film properties are shown in Fig.5-12. It can be seen from Fig.5-7 that film pressure, opening force and leakage increase first and then decrease with the increases of the depth of groove and pore. The maximum values of film pressure, opening force and leakage are in the model of $h_{g}=12 \mu \mathrm{m}$. The film pressure, opening force and leakage increase with the increases of speed. It can be seen from Fig. 8 that the ratio of stiffness to leakage decreases with the increases of speed. The higher ratio of 
stiffness to leakage is the model of $h_{g}=4 \mu \mathrm{m}$ when $n<2300 \mathrm{r} / \mathrm{min}$. However, The higher ratio of stiffness to leakage is the model of $h_{g}=20 \mu \mathrm{m}$ when $n>2300 \mathrm{r} / \mathrm{min}$.

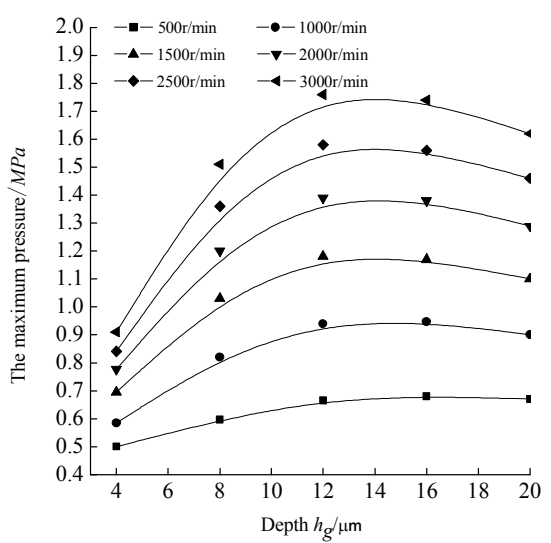

Fig.5 Effects of depth and $g$ speed on the maximum pressure

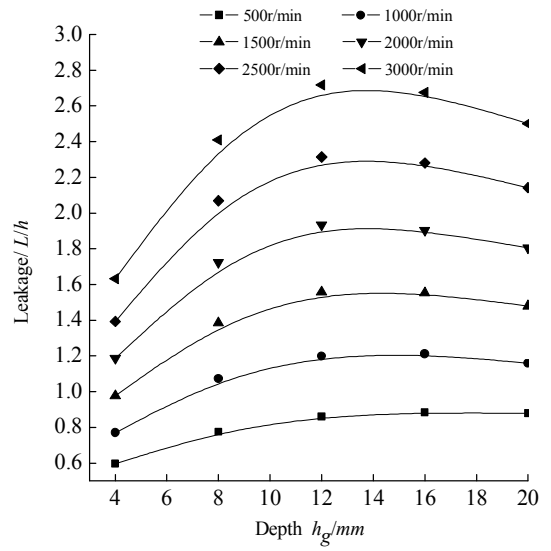

Fig.6 Effects of depth and speed on leakage
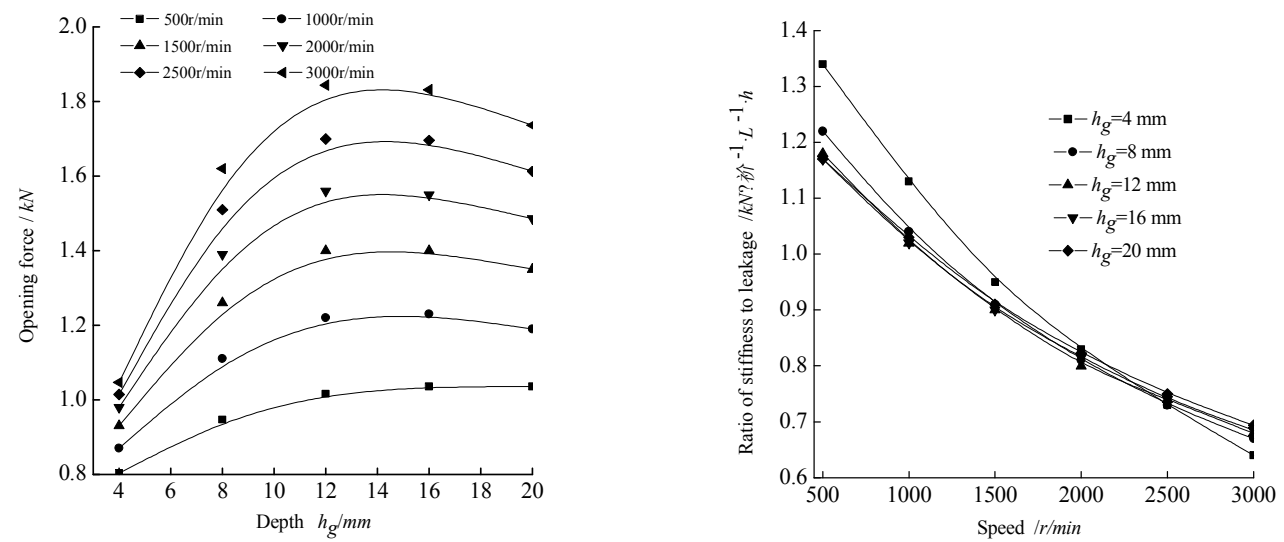

Fig.7 Effects of depth and speed on opening force Fig.8 Effects of depth and speed on ratio of stiffness to leakage

It can be seen from Fig.9-11 that film pressure, opening force and leakage increase first and then decrease with the increases of the depth of groove and pore. The maximum values of film pressure, opening force and leakage are in the model of $h_{g}=16 \mu \mathrm{m}$. The film pressure, the opening force and the leakage increase with the increases of medium pressure. It can be seen from Fig. 12 that the ratio of stiffness to leakage increases with the increases of medium pressure. The higher ratio of stiffness to leakage is the model of $h_{g}=20 \mu m$ when $p<1 M P a$. However, The higher ratio of stiffness to leakage is the model of $h_{g}=4 \mu m$ when $p>1 M P a$.

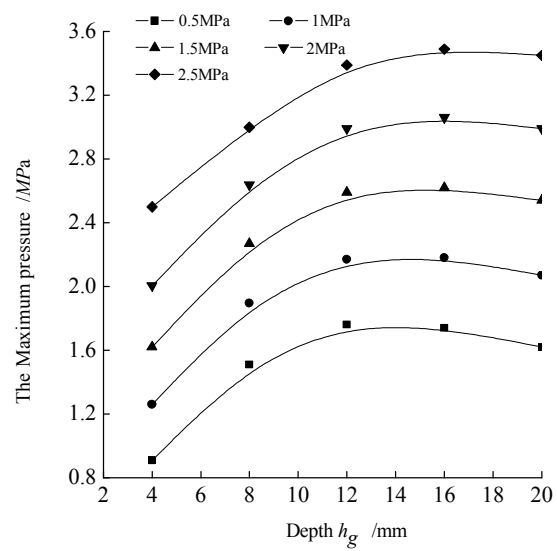

Fig.9 Effects of depth and medium pressure on the maximum pressure

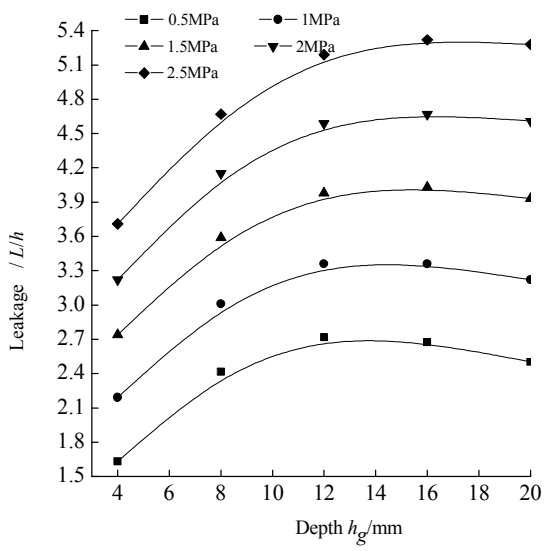

Fig.10 Effects of depth and medium pressure on the leakage 

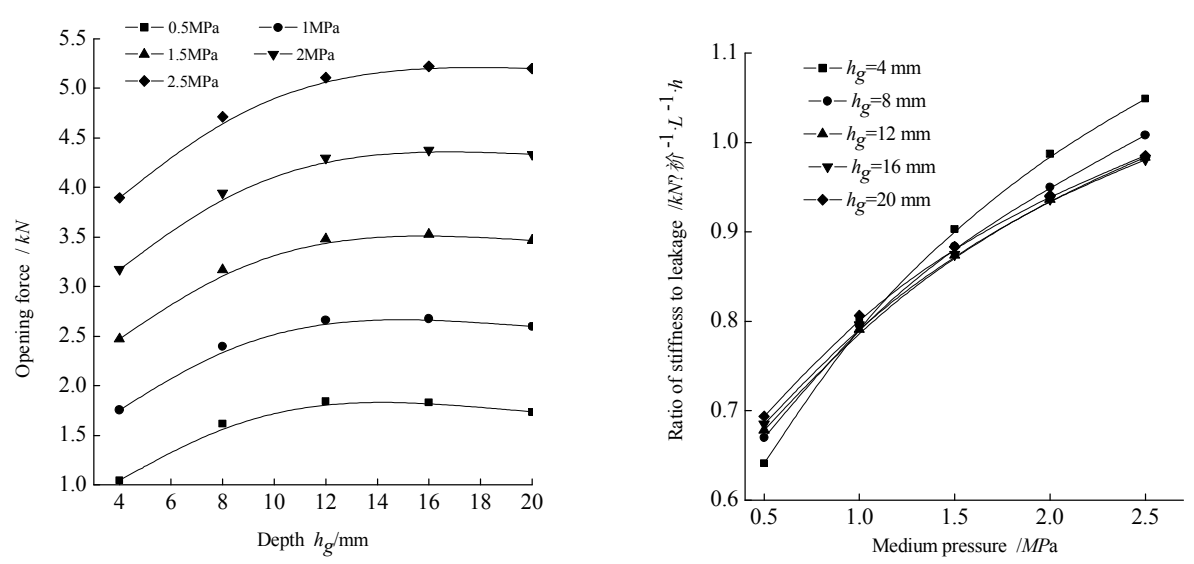

Fig.11 Effects of depth and medium pressure on the opening force stiffness to leakage

\section{Conclusions.}

(1) Dynamic pressure obtained in groove-pores coupled mechanical seal is higher than spiral groove mechanical seal, and the maximum pressure is at the root of spiral groove. (2) The film pressure, the opening force and the leakage increase first and then decrease with the increases of the depth of groove and pore. The higher ratio of stiffness to leakage is the model of $h_{g}=4 \mu m$ when $p>1 \mathrm{MPa}$ and $n<2300 \mathrm{r} / \mathrm{min}$. However, The higher ratio of stiffness to leakage is the model of $h_{g}=20 \mu m$ when $p<1 M P a$ and $n>2300 \mathrm{r} / \mathrm{min}$.

\section{References}

[1] Hiulong Chen, Zhibin Liu, Tong Liu, et al , J. Journal of Jiangsu University. 36 （2005 ） 43-47(In Chinese)

[2] Penggao Zhang, Boqin Gu, Long Wei, et al, J. Key Engineering Materials. 579-580(2014)548-553

[3] Huilong Cen, Wenyu. Li, Jianping Huang. et al, J. Lubrication Engineering. 37 (2012) 21-24(In Chinese)

[4] Yonghong Fu, Xinxiong Tang, Xijun. Hua, et al, J. Fluid Machinery. 38 （2010）1-4(In Chinese)

[5] Jianping Huang, Jiangsu University, Analysis on Inner Flow Field and Performance of Mechanical Seals With Spiral Groove and Micro-Cavities for Liquid. Zhenjiang, 2011(In Chinese)

[6] Penggao Zhang, Qiaofeng.Jiao, Biao Wang, et al, 5（2014）29-31(In Chinese) 